\title{
COMPLETELY REGULAR FUZZIFYING TOPOLOGICAL SPACES
}

\author{
A. K. KATSARAS \\ Received 24 March 2005 and in revised form 22 September 2005
}

Some of the properties of the completely regular fuzzifying topological spaces are investigated. It is shown that a fuzzifying topology $\tau$ is completely regular if and only if it is induced by some fuzzy uniformity or equivalently by some fuzzifying proximity. Also, $\tau$ is completely regular if and only if it is generated by a family of probabilistic pseudometrics.

\section{Introduction}

The concept of a fuzzifying topology was given in [1] under the name $L$-fuzzy topology. Ying studied in $[9,10,11]$ the fuzzifying topologies in the case of $L=[0,1]$. A classical topology is a special case of a fuzzifying topology. In a fuzzifying topology $\tau$ on a set $X$, every subset $A$ of $X$ has a degree $\tau(A)$ of belonging to $\tau, 0 \leq \tau(A) \leq 1$. In [4], we defined the degrees of compactness, of local compactness, Hausdorffnes, and so forth in a fuzzifying topological space $(X, \tau)$. We also introduced the fuzzifying proximities. Every fuzzifying proximity $\delta$ induces a fuzzifying topology $\tau_{\delta}$. In [6], we studied the level classical topologies $\tau^{\theta}, 0 \leq \theta<1$, corresponding to a fuzzifying topology $\tau$. In the same paper, we studied connectedness and local connectedness in fuzzifying topological spaces as well as the so-called sequential fuzzifying topologies. In [5], we introduced the fuzzifying syntopogenous structures. We also proved that every fuzzy uniformity $\mathcal{u}$, as it is defined by Lowen in [7], induces a fuzzifying proximity $\delta_{u}$, and that for every fuzzifying proximity $\delta$, there exists at least one fuzzy uniformity $U$ with $\delta=\delta_{\imath}$. Some of the results contained in papers $[4,6]$ are closely related to those which appeared in the papers $[12,13]$.

In this paper, we continue with the investigation of fuzzifying topologies. In particular, we study the completely regular fuzzifying topologies, that is, those fuzzifying topologies $\tau$ for which each level topology $\tau^{\theta}$ is completely regular. As in the classical case, we prove that for a fuzzifying topology $\tau$ on $X$, the following properties are equivalent: (1) $\tau$ is completely regular; (2) $\tau$ is uniformizable, that is, it is induced by some fuzzy uniformity; (3) $\tau$ is proximizable, that is, it is induced by some fuzzifying proximity; and (4) $\tau$ is generated by a family of so-called probabilistic pseudometrics on $X$. We also give a characterization of completely regular fuzzifying spaces in terms of continuous functions. Many Theorems on classical topologies follow as special cases of results obtained in the paper. 


\section{Preliminaries}

A fuzzifying topology on a set $X$ (see $[1,9,10,11]$ ) is a map $\tau: 2^{X} \rightarrow[0,1]$ (where $2^{X}$ is the power set of $X$ ) satisfying the following conditions:

(FT1) $\tau(X)=\tau(\varnothing)=1$;

(FT2) $\tau\left(A_{1} \cap A_{2}\right) \geq \tau\left(A_{1}\right) \wedge \tau\left(A_{2}\right)$;

(FT3) $\tau\left(\bigcup A_{i}\right) \geq \inf _{i} \tau\left(A_{i}\right)$.

If $\tau$ is a fuzzifying topology on $X$ and $x \in X$, then the $\tau$-neighborhood system of $x$ is the function

$$
N_{x}=N_{x}^{\tau}: 2^{X} \longrightarrow[0,1], \quad N_{x}(A)=\sup \{\tau(B): x \in B \subset A\} .
$$

By [9, Theorem 3.2], we have that $\tau(A)=\inf _{x \in A} N_{x}(A)$.

The following theorem is contained in [9] (see also [3, 13]).

Theorem 2.1. If $\tau$ is a fuzzifying topology on a set $X$, then the map $x \rightarrow N_{x}=N_{x}^{\tau}$, from $X$ to the fuzzy power set $\mathscr{F}\left(2^{X}\right)$ of $2^{X}$, has the following properties:

(FN1) $N_{x}(X)=1$ and $N_{x}(A)=0$ if $x \notin A$;

(FN2) $N_{x}\left(A_{1} \cap A_{2}\right)=N_{x}\left(A_{1}\right) \wedge N_{x}\left(A_{2}\right)$;

(FN3) $N_{x}(A) \leq \sup _{x \in D \subset A} \inf _{y \in D} N_{y}(D)$.

Conversely, if a map $x \rightarrow N_{x}$, from $X$ to $\mathscr{F}\left(2^{X}\right)$, satisfies (FN1)-(FN3), then the map

$$
\tau: 2^{X} \longrightarrow[0,1], \quad \tau(A)=\inf _{x \in A} N_{x}(A),
$$

is a fuzzifying topology and $N_{x}=N_{x}^{\tau}$ for every $x \in X$.

Let now $(X, \tau)$ be a fuzzifying topological space. To every subset $A$ of $X$ corrersponds a fuzzy subset $\bar{A}=\bar{A}^{\tau}$ of $X$ defined by $\bar{A}(x)=1-N_{x}\left(A^{c}\right)$ (see [13, Remark 3.16]). A function $f$, from a fuzzifying topological space $\left(X, \tau_{1}\right)$ to another one $\left(Y, \tau_{2}\right)$, is said to be continuous at some $x \in X$ (see $[4,13]$ ) if $N_{x}\left(f^{-1}(A)\right) \geq N_{f(x)}(A)$ for every subset $A$ of $Y$. If $f$ is continuous at every point of $X$, then it is said that $\left(\tau_{1}, \tau_{2}\right)$-continuous. As it is shown in [4], $f$ is continuous if and only if $\tau_{2}(A) \leq \tau_{1}\left(f^{-1}(A)\right)$ for every subset $A$ of $Y$. For $f: X \rightarrow Y$ a function and $\tau$ a fuzzifying topology on $Y, f^{-1}(\tau)$ is defined to be the weakest fuzzifying topology on $X$ for which $f$ is continuous. By [4], $f^{-1}(\tau)$ is given by the neighborhood structure $N_{x}(A)=N_{f(x)}\left(Y \backslash f\left(A^{c}\right)\right)$. If $\left(\tau_{i}\right)_{i \in I}$ is a family of fuzzifying topologies on $X$, we will denote by $\bigvee_{i \in I} \tau_{i}$, or by $\sup \tau_{i}$, the weakest of all fuzzifying topologies on $X$ which are finer than each $\tau_{i}$. As it is proved in [4], $\bigvee_{i \in I} \tau_{i}$ is given by the neighborhood structure

$$
N_{x}(A)=\sup \left\{\inf _{i \in J} N_{x}^{\tau_{i}}\left(A_{i}\right): x \in \bigcap_{i \in J} A_{i} \subset A\right\},
$$

where the infimum is taken over the family of all finite subsets $J$ of $I$ and all $A_{i} \subset X$, $i \in J$. For $Y$ a subset of a fuzzifying topological space $(X, \tau),\left.\tau\right|_{Y}$ will be the fuzzifying topology induced on $Y$ by $\tau$, that is, the fuzzifying topology $f^{-1}(\tau)$, where $f: Y \rightarrow X$ is the inclusion map. For a family $\left(X_{i}, \tau_{i}\right)_{i \in I}$ of fuzzifying topological spaces, the product 
fuzzifying topology $\tau=\prod \tau_{i}$ on $X=\prod X_{i}$ is the weakest fuzzifying topology on $X$ for which each projection $\pi_{i}: X \rightarrow X_{i}$ is continuous. Thus, $\tau=\bigvee_{i} \pi_{i}^{-1}\left(\tau_{i}\right)$ and it is given by the neighborhood structure

$$
N_{x}(A)=\sup \left\{\inf _{i \in J} N_{x_{i}}\left(A_{i}\right): x \in \bigcap_{i \in J} \pi_{i}^{-1}\left(A_{i}\right) \subset A\right\},
$$

where the supremum is taken over the family of all finite subsets $J$ of $I$ and $A_{i} \subset X_{i}$, for $i \in J$ (see [4]).

The degree of convergence to an $x \in X$, of a net $\left(x_{\delta}\right)$ in a fuzzifying topological space $(X, \tau)$, is the number $c\left(x_{\delta} \rightarrow x\right)=c^{\tau}\left(x_{\delta} \rightarrow x\right)$ defined by

$$
c\left(x_{\delta} \longrightarrow x\right)=\inf \left\{1-N_{x}(A): A \subset X,\left(x_{\delta}\right) \text { frequently in } A^{c}\right\} .
$$

As it is shown in [6], for $A \subset X$ and $x \in X$, we have

$$
\bar{A}(x)=\max \left\{c\left(x_{\delta} \longrightarrow x\right):\left(x_{\delta}\right) \text { net in } A\right\} .
$$

The degree of Hausdorffness of $X$ (see [4]) is defined by

$$
T_{2}(X)=1-\sup _{x \neq y} \sup \left\{c\left(x_{\delta} \longrightarrow x\right) \wedge c\left(x_{\delta} \longrightarrow y\right):\left(x_{\delta}\right) \text { net in } X\right\}
$$

Also, the degree of $X$ being $T_{1}$ is defined by

$$
T_{1}(X)=\inf _{x} \inf _{y \neq x} \sup \left\{N_{x}(B): y \notin B\right\}
$$

Let now $(X, \tau)$ be a fuzzifying topological space. For each $0 \leq \theta<1$, the family $B_{\theta}^{\tau}=\{A \subset$ $X: \tau(A)>\theta\}$ is a base for a classical topology $\tau^{\theta}$ on $X$ (see [5]). It is easy to see that a subset $B$ of $X$ is a $\tau^{\theta}$-neighborhood of $x$ if and only if $N_{x}(B)>\theta$. By [6], $T_{2}(X)$ (resp., $T_{1}(X)$ ) is the supremum of all $0 \leq \theta<1$ for which $\tau^{\theta}$ is $T_{2}$ (resp., $T_{1}$ ). Also, for $\tau=\vee \tau_{i}$, we have that $\tau^{\theta}=\sup _{i} \tau_{i}^{\theta}$ (see [6, Theorem 3.5]). If $\tau=\prod \tau_{i}$ is a product fuzzifying topology, then $\tau^{\theta}=\prod \tau_{i}^{\theta}$ (see [6, Theorem 3.5]). If $Y$ is a subspace of $(X, \tau)$ and $\tau_{1}=\tau \mid Y$, then $\tau_{1}^{\theta}=\tau^{\theta} \mid Y$. By [6, Theorem 3.10], for a fuzzifying topological space $(X, \tau), \operatorname{co}(X)$ coincides with the supremum of all $0<\theta<1$ for which $\tau^{1-\theta}$ is compact.

Next, we will recall the notion of a fuzzifying proximity given in [4]. A fuzzifying proximity on a set $X$ is a map $\delta: 2^{X} \times 2^{X} \rightarrow[0,1]$ satisfying the following conditions:

(FP1) $\delta(A, B)=1$ if the $A, B$ are not disjoint;

(FP2) $\delta(A, B)=\delta(B, A)$;

(FP3) $\delta(\varnothing, B)=0$;

(FP4) $\delta\left(A_{1} \cup A_{2}, B\right)=\delta\left(A_{1}, B\right) \vee \delta\left(A_{2}, B\right)$;

(FP5) $\delta(A, B)=\inf \left\{\delta(A, D) \vee \delta\left(D^{c}, B\right): D \subset X\right\}$. 
Every fuzzifying proximity $\delta$ induces a fuzzifying topology $\tau_{\delta}$ given by the neighborhood structure $N_{x}(A)=1-\delta\left(x, A^{c}\right)$. A fuzzifying proximity $\delta_{1}$ is said to be finer than another one $\delta_{2}$ if $\delta_{1}(A, B) \leq \delta_{2}(A, B)$ for all subsets $A, B$ of $X$. For $f: X \rightarrow Y$ a function and $\delta$ a fuzzifying proximity on $Y$, the function

$$
f^{-1}(\delta): 2^{X} \times 2^{X} \longrightarrow[0,1], \quad f^{-1}(\delta)(A, B)=\delta(f(A), f(B)),
$$

is a fuzzifying proximity on $X$ (see [4]) and it is the weakest of all fuzzifying proximities $\delta_{1}$ on $X$ for which $f$ is $\left(\delta_{1}, \delta\right)$-proximally continuous, that is, it satisfies $\delta_{1}(A, B) \leq$ $\delta(f(A), f(B))$ for all subsets $A, B$ of $X$. As it is shown in [4], $\tau_{f^{-1}(\delta)}=f^{-1}\left(\tau_{\delta}\right)$.

Let now $\left(\delta_{\lambda}\right)_{\lambda \in \Lambda}$ be a family of fuzzifying proximities on a set $X$. We will denote by $\delta=\bigvee_{\lambda} \delta_{\lambda}$, or by $\sup \delta_{\lambda}$, the weakest fuzzifying proximity on $X$ which is finer than each $\delta_{\lambda}$. By $[4$, Theorem 8.10$], \delta$ is given by

$$
\delta(A, B)=\inf \left\{\sup _{i, j} \inf _{\lambda \in \Lambda} \delta_{\lambda}\left(A_{i}, B_{j}\right)\right\},
$$

where the infimum is taken over all finite collections $\left(A_{i}\right),\left(B_{j}\right)$ of subsets of $X$ with $A=$ $\bigcup A_{i}, B=\bigcup B_{j}$. Moreover, $\tau_{\delta}=\bigvee \tau_{\delta_{\lambda}}$ (see [4]).

Finally, we will recall the definition of a fuzzy uniformity introduced by Lowen in [7]. For a set $X$, let $\Omega_{X}$ be the collection of all functions $\alpha: X \times X \rightarrow[0,1]$ such that $\alpha(x, x)=1$ for all $x \in X$. For $\alpha, \beta \in \Omega_{X}$, the $\alpha \wedge \beta, \alpha \circ \beta$ and $\alpha^{-1}$ are defined by $\alpha \wedge \beta(x, y)=$ $\alpha(x, y) \wedge \beta(x, y), \alpha \circ \beta(x, y)=\sup _{z} \beta(x, z) \wedge \alpha(z, y), \alpha^{-1}(x, y)=\alpha(y, x)$. If $\alpha=\alpha^{-1}$, then $\alpha$ is called symmetric. A fuzzy uniformity on $X$ is a nonempty subset $U$ of $\Omega_{X}$ satisfying the following conditions.

(FU1) If $\alpha, \beta \in \mathcal{U}$, then $\alpha \wedge \beta \in \mathcal{U}$.

(FU2) If $\alpha \in \mathcal{U}$ is such that, for every $\epsilon>0$, there exists a $\beta \in \mathcal{U}$ with $\beta \leq \alpha+\epsilon$, then $\alpha \in \mathcal{U}$.

(FU3) For each $\alpha \in \mathcal{U}$ and each $\epsilon>0$, there exists a $\beta \in \mathcal{U}$ with $\beta \circ \beta \leq \alpha+\epsilon$.

(FU4) If $\alpha \in \mathcal{U}$, then $\alpha^{-1} \in \mathcal{U}$.

A subset $\mathscr{B}$, of a fuzzy uniformity $\mathcal{U}$, is a base for $\mathcal{U}$ if for each $\alpha \in \mathcal{U}$ and each $\epsilon>0$, there exists $\beta \in \mathscr{B}$ with $\beta \leq \alpha+\epsilon$. It is easy to see that for a subset $\mathscr{B}$ of $\Omega_{X}$, the following are equivalent.

(1) $\mathscr{B}$ is a base for a fuzzy uniformity on $X$.

(2) (a) If $\alpha, \beta \in \mathscr{S}$ and $\epsilon>0$, then there exists $\gamma \in \mathscr{B}$ with $\gamma \leq \alpha \wedge \beta+\epsilon$.

(b) For each $\alpha \in \mathscr{B}$ and each $\epsilon>0$, there exists $\beta \in \mathscr{H}$ with $\beta \circ \beta \leq \alpha+\epsilon$.

(c) For each $\alpha \in \mathscr{B}$ and each $\epsilon>0$, there exists $\beta \in \mathscr{B}$ with $\beta \leq \alpha^{-1}+\epsilon$.

In case (2) is satisfied, the fuzzy uniformity $\mathcal{U}$ for which $\mathscr{B}$ is a base consists of all $\alpha \in \Omega_{X}$ such that for each $\epsilon>0$, there exists a $\beta \in \mathscr{B}$ with $\beta \leq \alpha+\epsilon$.

By [5], every fuzzy uniformity $\mathcal{U}$ on $X$ induces a fuzzifying proximity $\delta_{u}$ defined by

$$
\delta_{u}(A, B)=\inf _{\alpha \in \mathcal{U}} \sup _{x \in A, y \in B} \alpha(x, y) .
$$


In case $\mathscr{B}$ is a base for $U$, then

$$
\delta_{u}(A, B)=\inf _{\alpha \in \mathscr{B}} \sup _{x \in A, y \in B} \alpha(x, y) .
$$

Every fuzzy uniformity $\mathcal{U}$ induces a fuzzifying topology $\tau_{\vartheta}$ given by the neighborhood structure

$$
N_{x}(A)=1-\delta_{U}\left(x, A^{c}\right)=1-\inf _{\alpha \in U} \sup _{y \notin A} \alpha(x, y)
$$

For every fuzzifying proximity $\delta$, there exists at least one compatible fuzzy uniformity, that is, a fuzzy uniformity $U$ with $\delta_{u}=\delta$ (see [5, Theorem 11.4]).

\section{Probabilistic pseudometrics}

A fuzzy real number is a fuzzy subset $u$ of the real numbers $\mathbb{R}$ which is increasing, left continuous, and such that $\lim _{t \rightarrow+\infty} u(t)=1, \lim _{t \rightarrow-\infty} u(t)=0$. A fuzzy real number $u$ is said to be nonnegative if $u(t)=0$ if $t \leq 0$. We will denote by $\mathbb{R}_{\phi}^{+}$the collection of all nonnegative fuzzy real numbers. To every real number $r$ corresponds a fuzzy real number $\bar{r}$, where $\bar{r}(t)=0$ if $t \leq r$ and $\bar{r}(t)=1$ if $t>r$. For $u, v \in \mathbb{R}_{\phi}^{+}$, we define $u \preceq v$ if and only if $v(t) \leq u(t)$ for all $t \in \mathbb{R}$. If $\mathscr{A}$ is a nonempty subset of $\mathbb{R}_{\phi}^{+}$and if $u_{o} \in \mathbb{R}_{\phi}^{+}$is defined by $u_{o}(t)=\sup _{v \in \mathscr{A}} v(t)$, then $u_{o}$ is the biggest of all $u \in \mathbb{R}_{\phi}^{+}$with $u \preceq v$ for all $v \in \mathscr{A}$. We will denote $u_{o}$ by inf $\mathscr{A}$ or by $\bigwedge \mathscr{A}$. For $u_{1}, u_{2} \in \mathbb{R}_{\phi}^{+}$, we define $u=u_{1} \oplus u_{2} \in \mathbb{R}_{\phi}^{+}$by $u(t)=$ $\sup \left\{u_{1}\left(t_{1}\right) \wedge u_{2}\left(t_{2}\right): t=t_{1}+t_{2}\right\}$. Also, for $u \in \mathbb{R}_{\phi}^{+}$and $\lambda>0$, we define $\lambda u$ by $(\lambda u)(t)=$ $u\left(\lambda^{-1} t\right)$. It is easy to see that for $u \in \mathbb{R}_{\phi}^{+}$and $\lambda>0$, we have $(\bar{\lambda} \oplus u)(t)=u(t-\lambda)$.

Definition 3.1. A probabilistic pseudometric on a set $X$ (see [2]) is a mapping $F: X \times X \rightarrow$ $\mathbb{R}_{\phi}^{+}$such that for all $x, y, z \in X$,

$$
F(x, x)=\overline{0}, \quad F(x, y)=F(y, x), \quad F(x, z) \preceq F(x, y) \oplus F(y, z) .
$$

If in addition $F(x, y)(0+)=0$ when $x \neq y$, then $F$ is called a probabilistic metric.

If $r_{1}, r_{2}$ are nonnegative real numbers, then $\overline{r_{1}} \preceq \overline{r_{2}}$ if and only if $r_{1} \leq r_{2}$. Also, for $r=\left|r_{1}-r_{2}\right|$, we have that

$$
\bar{r}=\wedge\left\{u \in \mathbb{R}_{\phi}^{+}: \overline{r_{2}} \preceq u \oplus \overline{r_{1}}, \overline{r_{1}} \preceq u \oplus \overline{r_{2}}\right\}
$$

In fact, let $u_{o}=\wedge\left\{u \in \mathbb{R}_{\phi}^{+}: \overline{r_{2}} \preceq u \oplus \overline{r_{1}}\right.$ and $\left.\overline{r_{1}} \preceq u \oplus \overline{r_{2}}\right\}$ and assume that (say) $r_{1} \geq r_{2}$. Let $u \in \mathscr{R}_{\phi}^{+}$be such that $\overline{r_{2}} \preceq u \oplus \overline{r_{1}}, \overline{r_{1}} \preceq u \oplus \overline{r_{2}}$. Then $\overline{r_{1}}(t) \geq\left(u \oplus \overline{r_{2}}\right)(t)=u\left(t-r_{2}\right)$ for all $t$. If $s<r_{1}$, then $0=\overline{r_{1}}(s) \geq u\left(s-r_{2}\right)$ and so $u\left(r_{1}-r_{2}\right)=\sup _{s<r_{1}} u\left(s-r_{2}\right)=0$ which implies that $\bar{r} \preceq u$. Thus $\bar{r} \preceq u_{0}$. On the other hand, we have $\bar{r} \oplus \overline{r_{2}}=\overline{r_{1}}$ and $\bar{r} \oplus \overline{r_{1}}=\overline{2 r_{1}-r_{2}}$. Since $\overline{r_{2}} \preceq \overline{2 r_{1}-r_{2}}$, it follows that $u_{o} \preceq \bar{r}$, and hence $\bar{r}=u_{o}$. Motivated by the above, we define the following distance function on $\mathscr{R}_{\phi}^{+}$:

$$
D: \mathscr{R}_{\phi}^{+} \times \mathscr{R}_{\phi}^{+} \longrightarrow \mathscr{R}_{\phi}^{+}, \quad D\left(u_{1}, u_{2}\right)=\wedge\left\{u \in \mathscr{R}_{\phi}^{+}: u_{1} \preceq u_{2} \oplus u, u_{2} \preceq u \oplus u_{1}\right\}
$$


Then $D$ is a probabilistic pseudometric on $\mathscr{R}_{\phi}^{+}$. In fact, it is clear that $D\left(u_{1}, u_{2}\right)=$ $D\left(u_{2}, u_{1}\right)$. Also, since $u=u \oplus \overline{0}$, when $u \in \mathscr{R}_{\phi}^{+}$, we have that $D(u, u)=\overline{0}$. Finally, let $D\left(u_{1}, u_{2}\right)\left(t_{1}\right) \wedge D\left(u_{2}, u_{3}\right)\left(t_{2}\right)>\theta>0$. There are $v_{1}, v_{2} \in \mathscr{R}_{\phi}^{+}$with $u_{1} \preceq v_{1} \oplus u_{2}, u_{2} \preceq v_{1} \oplus$ $u_{1}, u_{3} \preceq v_{2} \oplus u_{2}, u_{2} \preceq v_{2} \oplus u_{3}, v_{1}\left(t_{1}\right)>\theta, v_{2}\left(t_{2}\right)>\theta$. Now $u_{1} \preceq v_{1} \oplus u_{2} \preceq v_{1} \oplus\left(v_{2} \oplus u_{3}\right)=$ $\left(v_{1} \oplus v_{2}\right) \oplus u_{3}$ and $u_{3} \preceq v_{2} \oplus u_{2} \preceq v_{2} \oplus\left(v_{1} \oplus u_{1}\right)=\left(v_{1} \oplus v_{2}\right) \oplus u_{1}$. Thus, $D\left(u_{1}, u_{3}\right) \preceq v_{1} \oplus$ $v_{2}$ and $D\left(u_{1}, u_{3}\right)\left(t_{1}+t_{2}\right) \geq v_{1}\left(t_{1}\right) \wedge v_{2}\left(t_{2}\right)>\theta$. This proves that $D\left(u_{1}, u_{3}\right) \preceq D\left(u_{1}, u_{2}\right) \oplus$ $D\left(u_{2}, u_{3}\right)$ and the claim follows. We will refer to $D$ as the usual probabilistic pseudometric on $\mathscr{R}_{\phi}^{+}$.

Let now $F$ be a probabilistic pseudometric on $X$. For $t>0$, let $u_{F, t}$ be defined on $X^{2}$ by $u_{F, t}(x, y)=F(x, y)(t)$. The family $\mathscr{B}_{F}=\left\{u_{F, t}: t>0\right\}$ is a base for a fuzzy uniformity $u_{F}$ on $X$. Let $\tau_{F}$ be the fuzzifying topology induced by $u_{F}$.

In the rest of the paper, we will consider on $\mathscr{R}_{\phi}^{+}$the fuzzifying topology induced by the usual probabilistic pseudometric $D$.

Theorem 3.2. A probabilistic pseudometric $F$, on a fuzzifying topological space $(X, \tau)$, is $\tau \times \tau$ continuous if and only if $\tau_{F} \leq \tau$.

Proof. Assume that $\tau_{F} \leq \tau$ and let $G$ be a subset of $\mathbb{R}_{\phi}^{+}$and $u=F\left(x_{o}, y_{o}\right)$ with $N_{u}(G)>\theta>$ 0 . There exists a $t>0$ such that $1-\sup _{v \notin G} D(v, u)(t)>\theta$. For $x, y \in X$, we have

$$
F(x, y) \preceq F\left(x, x_{o}\right) \oplus\left(x_{o}, y_{o}\right) \oplus F\left(y_{o}, y\right)=\left[F\left(x, x_{o}\right) \oplus F\left(y, y_{o}\right)\right] \oplus F\left(x_{o}, y_{o}\right) .
$$

Similarly, $F\left(x_{o}, y_{o}\right) \preceq\left[F\left(x, x_{o}\right) \oplus F\left(y, y_{o}\right)\right] \oplus F(x, y)$. Thus,

$$
D\left(F(x, y), F\left(x_{o}, y_{o}\right)\right) \preceq F\left(x, x_{o}\right) \oplus F\left(y, y_{o}\right) .
$$

Let

$$
A_{1}=\left\{x \in X: F\left(x, x_{o}\right)\left(\frac{t}{2}\right) \geq 1-\theta\right\}, \quad A_{2}=\left\{x \in X: F\left(y, y_{o}\right)\left(\frac{t}{2}\right) \geq 1-\theta\right\} .
$$

If $x \in A_{1}, y \in A_{2}$, then

$$
D\left(F(x, y), F\left(x_{o}, y_{o}\right)\right)(t) \geq F\left(x, x_{o}\right)\left(\frac{t}{2}\right) \wedge F\left(y, y_{o}\right)\left(\frac{t}{2}\right) \geq 1-\theta,
$$

and so $F(x, y) \in G$. Also, $N_{x_{o}}^{\tau}\left(A_{1}\right) \geq N_{x_{o}}^{\tau_{F}}\left(A_{1}\right) \geq 1-\sup _{x \notin A_{1}} F\left(x, x_{o}\right)(t / 2) \geq \theta$ and $N_{y_{o}}^{\tau}\left(A_{2}\right)$ $\geq \theta$. Therefore,

$$
N_{\left(x_{0}, y_{o}\right)}^{\tau \times \tau}\left(F^{-1}(G)\right) \geq N_{x_{o}}^{\tau}\left(A_{1}\right) \wedge N_{y_{o}}^{\tau}\left(A_{1}\right) \geq \theta
$$

which proves that $N_{\left(x_{0}, y_{0}\right)}^{\tau \times \tau}\left(F^{-1}(G)\right) \geq N_{F\left(x_{o}, y_{o}\right)}(G)$ and so $F$ is $\tau \times \tau$ continuous. Conversely, assume that $F$ is $\tau \times \tau$ continuous and let $N_{x_{o}}^{\tau_{F}}(A)>\theta>0$. Choose $\epsilon>0$ such that $N_{x_{o}}^{\tau_{F}}(A)>\theta+\epsilon$. There exists a $t>0$ such that $1-\sup _{x \notin A} F\left(x, x_{0}\right)(t)>\theta+\epsilon$. If

$$
Z=\left\{u \in \mathbb{R}_{\phi}^{+}: D(u, \overline{0})(t)=u(t)>1-\theta-\epsilon\right\},
$$

then

$$
N_{\overline{0}}(Z) \geq 1-\sup _{u \notin Z} D(u, \overline{0})(t) \geq \theta+\epsilon>\theta
$$


Since $F$ is $\tau \times \tau$ continuous and $F\left(x_{0}, x_{0}\right)=\overline{0}$, there exists a subset $A_{1}$ of $X$ containing $x_{o}$ such that $A_{1} \times A_{1} \subset F^{-1}(Z)$ and $N_{x_{0}}\left(A_{1}\right)>\theta$. If $x \in A_{1}$, then $F\left(x, x_{o}\right) \in Z$ and so $F\left(x, x_{o}\right)(t)>1-\theta-\epsilon$, which implies that $x \in A$. Thus, $A_{1} \subset A$ and so $N_{x_{o}}(A) \geq N_{x_{o}}^{\tau_{F}}(A)$ for every subset $A$ of $X$ and every $x_{o} \in X$. Hence, $\tau_{F} \leq \tau$ and the result follows.

Theorem 3.3. Let $F$ be a probabilistic pseudometric on a set $X, \tau=\tau_{F},\left(x_{\delta}\right)_{\delta \in \Delta}$ a net in $X$, and $x \in X$. Then

$$
c\left(x_{\delta} \longrightarrow x\right)=\inf _{t>0} \liminf _{\delta} F\left(x_{\delta}, x\right)(t)
$$

Proof. Let $d=\inf _{t>0} \liminf _{\delta} F\left(x_{\delta}, x\right)(t)$ and assume that $d<\theta<1$. There exists a $t>0$ such that $\liminf _{\delta} F\left(x_{\delta}, x\right)(t)<\theta$. Let $A=\{y: F(y, x)(t)>\theta\}$. Then $\left(x_{\delta}\right)$ is not eventually in $A$, and so $c\left(x_{\delta} \rightarrow x\right) \leq 1-N_{x}(A) \leq \sup _{y \notin A} F(y, x)(t) \leq \theta$, which proves that $c\left(x_{\delta} \rightarrow\right.$ $x) \leq d$. On the other hand, let $c\left(x_{\delta} \rightarrow x\right)<r<1$. There exists a subset $B$ of $X$ such that $\left(x_{\delta}\right)$ is not eventually in $B$ and $1-N_{x}(B)<r$. Let $s>0$ be such that $1-\sup _{y \notin B} F(y, x)(s)>1-$ $r$. For each $\delta \in \Delta$, there exists $\delta^{\prime} \geq \delta$ with $x_{\delta^{\prime}} \notin B$, and so $F\left(x_{\delta^{\prime}}, x\right)(s) \leq \sup _{y \notin B} F(y, x)(s)$. Thus, $d \leq \liminf _{\delta} F\left(x_{\delta}, x\right)(s)<r$, which proves that $d \leq c\left(x_{\delta} \rightarrow x\right)$ and the result follows.

Theorem 3.4. Let $F_{1}, F_{2}, \ldots, F_{n}$ be probabilistic pseudometrics on $X$ and define $F$ by

$$
F(x, y)(t)=\min _{1 \leq k \leq n} F_{k}(x, y)(t)
$$

Then $F$ is a probabilistic pseudometric and $\tau_{F}=\bigvee_{k+1}^{n} \tau_{F_{k}}$.

Proof. Using induction on $n$, it suffices to prove the result in the case of $n=2$. It follows easily that $F$ is a probabilistic pseudometric. Since $F_{1}, F_{2} \preceq F$, it follows that $\tau_{F_{1}}, \tau_{F_{2}} \leq \tau_{F}$ and so $\tau_{o}=\tau_{F_{1}} \vee \tau_{F_{2}} \leq \tau_{F}$. On the other hand, let $N_{x}^{\tau_{F}}(A)>\theta>0$. There exists a $t>0$ such that $1-\sup _{y \notin A} F(y, x)(t)>\theta$. Let $B_{i}=\left\{y \in A^{c}: F_{i}(y, x)(t)<1-\theta\right\}, i=1,2$. Then $A^{c}=$ $B_{1} \cup B_{2}$ and so $A=A_{1} \cap A_{2}, A_{i}=B_{i}^{c}$. Moreover $N_{x}^{\tau_{F_{i}}}\left(A_{i}\right) \geq 1-\sup _{y \in B_{i}} F_{i}(y, x)(t) \geq \theta$, and thus

$$
N_{x}^{\tau_{o}}(A) \geq N_{x}^{\tau_{o}}\left(A_{1}\right) \bigwedge N_{x}^{\tau_{o}}\left(A_{2}\right) \geq N_{x}^{\tau_{F_{1}}}\left(A_{1}\right) \bigwedge N_{x}^{\tau_{F_{2}}}\left(A_{2}\right) \geq \theta
$$

This proves that $N_{x}^{\tau_{o}}(A) \geq N_{x}^{\tau_{F}}(A)$ and the result follows.

For $\mathscr{F}_{F}$ a family of probabilistic pseudometrics on a set $X$, we will denote by $\tau_{\mathscr{F}}$ the supremum of the fuzzifying topologies $\tau_{F}, F \in \mathscr{F}_{\mathscr{F}}$, that is, $\tau_{\mathscr{F}}=\bigvee_{F \in \mathscr{F}} \tau_{F}$.

THEOREM 3.5. If $\tau=\tau_{\mathscr{F}}$, where $\mathscr{F}$ is a family of probabilistic pseudometrics on a set $X$, then $T_{2}(X)=T_{1}(X)=1-\sup _{y \neq x} \inf _{F \in \mathscr{F}} F(x, y)(0+)$.

Proof. Let $d=1-\sup _{y \neq x} \inf _{F \in \mathscr{F}} F(x, y)(0+)$. It is always true that $T_{2}(X) \leq T_{1}(X)$. Suppose that $T_{1}(X)>r>0$ and let $x \neq y$. Since $\tau^{r}$ is $T_{1}$, there exists a $\tau^{r}$-neighborhood $A$ of $x$ not containing $y$. Now $N_{x}(A)>r$, and hence there are subsets $A_{1}, \ldots, A_{n}$ of $X$ and $F_{1}, \ldots, F_{n} \in \mathscr{F}_{\text {s }}$ such that $\bigcap A_{k} \subset A, N_{x}^{\tau_{F_{k}}}\left(A_{k}\right)>r$. Since $y$ is not in $A$, there exists a $k$ with 
$y \notin A_{k}$. Let $t>0$ be such that

$$
1-\sup _{z \notin A_{k}} F_{k}(z, x)(t)>r \text { and so } \inf _{F \in \mathscr{F}} F(x, y)(t)(0+) \leq F_{k}(x, y)(t)<1-r,
$$

which proves that $d \geq r$. Thus $d \geq T_{1}(X)$. On the other hand, assume that $d>\theta>0$ and let $x \neq y$. Choose $\epsilon>0$ such that $d>\theta+\epsilon$. There exists $F \in \mathscr{F}$ with $F(x, y)(0+)<1-\theta-$ $\epsilon$, and hence $F(x, y)(t)<1-\theta-\epsilon$ for some $t>0$. Let

$$
A=\left\{z: F(z, x)\left(\frac{t}{2}\right)>1-\theta-\epsilon\right\}, \quad B=\left\{z: F(z, y)\left(\frac{t}{2}\right)>1-\theta-\epsilon\right\} .
$$

Clearly $x \in A, y \in B$. If $z \in A \cap B$, then

$$
F(x, y)(t) \geq F(x, z)\left(\frac{t}{2}\right) \wedge F(z, y)\left(\frac{t}{2}\right)>1-\theta-\epsilon,
$$

a contradiction. Thus $A \cap B=\varnothing$. Moreover

$$
N_{x}(A) \geq N_{x}^{\tau_{F}}(A) \geq 1-\sup _{z \notin A} F(x, z)\left(\frac{t}{2}\right) \geq \theta+\epsilon>\theta, \quad N_{y}(A)>\theta .
$$

It follows that $T_{2}(X) \geq d$ and the proof is complete.

Let us say that a fuzzifying topology $\tau$ on a set $X$ is pseudometrizable if there exists a probabilistic pseudometric $F$ on $X$ with $\tau=\tau_{F}$.

Theorem 3.6. A fuzzifying topology $\tau$ on $X$ is pseudometrizable if and only if each level topology $\tau^{\theta}, 0 \leq \theta<1$, is pseudometrizable.

Proof. Assume that $\tau=\tau_{F}$ for some probabilistic pseudometric $F$ and let $0 \leq \theta<1$. For each positive integer $n$, with $n>1 /(1-\theta)$, let

$$
A_{n}=\left\{(x, y) \in X^{2}: F(x, y)\left(\frac{1}{n}\right)>1-\theta-\frac{1}{n}\right\}
$$

Then $A_{n+1} \subset A_{n}$ and the family $\mathscr{D}=\left\{A_{n}: n \in \mathbb{N}, n>1 /(1-\theta)\right\}$ is a base for a uniformity $\mathcal{U}$ on $X$. The topology $\sigma_{\theta}$ induced by $\mathcal{U}$ is pseudometrizable since $\mathscr{D}$ is countable. Moreover $\sigma_{\theta}=\tau^{\theta}$. Indeed, let $A$ be a $\sigma_{\theta}$-neighborhood of $x$. There exists $n \in \mathbb{N}, n>1 /(1-\theta)$, such that $B=\{y: F(x, y)(1 / n)>1-\theta-1 / n\} \subset A$. Now

$$
N_{x}^{\tau}(A) \geq N_{x}^{\tau}(B) \geq 1-\sup _{y \notin B} F(x, y)\left(\frac{1}{n}\right) \geq \theta+\frac{1}{n}>\theta,
$$

and so $A$ is a $\tau^{\theta}$-neighborhood of $x$. Conversely, assume that $A$ is a $\tau^{\theta}$-neighborhood of $x$. There exists $\epsilon>0$ with $N_{x}(A)>\theta+\epsilon$. Now there exists a positive integer $n>1 / \epsilon$ such 
that $1-\sup _{y \notin A} F(x, y)(1 / n)>\theta+1 / n$. Hence

$$
\left\{y: F(x, y)\left(\frac{1}{n}\right)>1-\theta-\frac{1}{n}\right\} \subset A,
$$

which implies that $A$ is a $\sigma_{\theta}$-neighborhood of $x$. Thus $\tau^{\theta}=\sigma_{\theta}$, and therefore each $\tau^{\theta}$ is pseudometrizable. Conversely, suppose that each $\tau^{\theta}$ is pseudometrizable. By an argument analogous to the one used in the proof of [6, Theorem 3.3], we show that there exists a family $\left\{d_{\theta}: 0 \leq \theta<1\right\}$ of pseudometrics on $X$ such that $d_{\theta}=\sup _{\theta_{1}>\theta} d_{\theta_{1}}$, for each $0 \leq \theta<1$, and $\tau^{\theta}$ coincides with the topology induced by the pseudometric $d_{\theta}$. Now, for $x, y \in X$, define $F(x, y): \mathbb{R} \rightarrow[0,1]$ by $F(x, y)(t)=0$ if $t \leq 0$ and $F(x, y)(t)=\sup \{\theta$ : $\left.0<\theta \leq 1, d_{1-\theta}(x, y)<t\right\}$ if $t>0$. It is clear that $F(x, y)$ is increasing and left continuous. For $0<r<1$ and $t>d_{1-r}(x, y)$, we have that $F(x, y)(t) \geq r$, and so $\lim _{t \rightarrow \infty} F(x, y)(t)=1$. Also $F(x, x)(t)=1$ for every $x$ and every $t>0$. To show that $F$ is a probabilistic pseudometric on $X$, we must prove that it satisfies the triangle inequality. So, let $F(x, y)\left(t_{1}\right) \wedge$ $F(y, z)\left(t_{2}\right)>\theta>0$. Then $d_{1-\theta}(x, y)<t_{1}, d_{1-\theta}(y, z)<t_{2}$, and so $d_{1-\theta}(x, z)<t_{1}+t_{2}$, which implies that $F(x, z)\left(t_{1}+t_{2}\right) \geq \theta$. Thus the triangle inequality is satisfied and $F$ is a probabilistic pseudometric. We will finish the proof by showing that $\tau_{F}=\tau$. So let $N_{x}^{\tau_{F}}>\theta>0$ and choose $t>0$ such that $1-\sup _{y \notin A} F(y, x)(t)>\theta$. If now $d_{\theta}(x, y)<t$, then $F(x, y)(t) \geq$ $1-\theta$, and thus $y \in A$, which proves that $A$ is a $\sigma_{\theta}=\tau^{\theta}$ neighborhood of $x$. Hence $\tau \geq \tau_{F}$. On the other hand, let $B$ be a $\tau^{\theta}$-neighborhood of $x$. There exists $\theta_{1}>\theta$ such that $N_{x}(B)>$ $\theta_{1}$. Now $B$ is a $\tau_{\theta_{1}}$-neighborhood of $x$, and so there exists $t>0$ such that $\left\{y: d_{\theta_{1}}(x, y)<\right.$ $t\} \subset B$. If $F(x, y)(t)>1-\theta_{1}$, then there exists $\alpha>1-\theta_{1}$ such that $d_{1-\alpha}(x, y)<t$ and so $d_{\theta_{1}}(x, y)<t$. Thus $\left\{y: F(x, y)(t)>1-\theta_{1}\right\} \subset B$, and therefore

$$
N_{x}^{\tau_{F}}(B) \geq 1-\sup _{y \notin B} F(x, y)(t) \geq \theta_{1}>\theta .
$$

Thus, $\tau_{F} \geq \tau$ and the result follows.

Theorem 3.7. Let $(X, F)$ be a probabilistic pseudometric space, $A \subset X$, and $x \in X$.

Let

$$
\begin{aligned}
& \alpha=\sup \left\{\inf _{t>0} \liminf _{n} F\left(x_{n}, x\right)(t):\left(x_{n}\right) \text { sequence in } A\right\}, \\
& \beta=\sup \left\{\liminf _{n} F\left(x_{n}, x\right)\left(t_{n}\right): t_{n} \longrightarrow 0+,\left(x_{n}\right) \text { sequence in } A\right\}, \\
& \gamma=\sup \left\{\liminf _{n} F\left(x_{n}, x\right)(1 / n):\left(x_{n}\right) \text { sequence in } A\right\} .
\end{aligned}
$$

Then $\alpha=\beta=\gamma=\bar{A}(x)$.

Proof. If $\left(x_{n}\right) \subset A$, then

$$
\bar{A}(x) \geq c\left(x_{n} \longrightarrow x\right)=\inf _{t>0} \liminf _{n} F\left(x_{n}, x\right)(t),
$$

and so $\bar{A}(x) \geq \alpha$. Assume that $\beta>\theta>0$. There exist a sequence $\left(x_{n}\right) \in A$ and a sequence $\left(t_{n}\right)$ of positive real numbers, with $t_{n} \rightarrow 0+$, such that $\liminf _{n} F\left(x_{n}, x\right)\left(t_{n}\right)>\theta$. Let $t>0$ 
and choose $k$ such that $t_{n}<t$ when $n \geq k$. For $m \geq k$, we have $\inf _{n>m} F\left(x_{n}, x\right)(t) \geq$ $\inf _{n \geq m} F\left(x_{n}, x\right)\left(t_{n}\right)>\theta$. Thus $\liminf _{n} F\left(x_{n}, x\right)(t)>\theta$ for each $t>0$ and so $\alpha \geq \theta$, which proves that $\alpha \geq \beta$. Clearly $\beta \geq \gamma$. Finally, $N_{x}\left(A^{c}\right) \geq 1-\sup _{y \in A} F(y, x)(1 / n)$, and so $\sup _{y \in A} F(y, x)(1 / n) \geq 1-N_{x}\left(A^{c}\right)=\bar{A}(x)>\bar{A}(x)-1 / n$. Hence, for each $n \in \mathbb{N}$, there exists $x_{n} \in A$ with $F\left(x_{n}, x\right)(1 / n)>\bar{A}(x)-1 / n$. Consequently,

$$
\gamma \geq \liminf _{n} F\left(x_{n}, x\right)\left(\frac{1}{n}\right) \geq \liminf _{n}\left(\bar{A}(x)-\frac{1}{n}\right)=\bar{A}(x),
$$

and so $\gamma \geq \bar{A}(x) \geq \alpha \geq \beta \geq \gamma$, which completes the proof.

In view of [6, Theorem 4.14], we have the following corollary.

Corollary 3.8. Every pseudometrizable fuzzifying topological space is $\mathbb{N}$-sequential and hence sequential.

THeORem 3.9. If $\left(F_{n}\right)$ is a sequence of probabilistic pseudometrics on a set $X$, then there exists a probabilistic pseudometric F such that $\tau_{F}=\bigvee_{n} \tau_{F_{n}}$.

Proof. If $F$ is a probabilistic pseudometric on $X$ and if $\bar{F}$ is defined by $\bar{F}(x, y)(t)=$ $F(x, y)(t)$ if $t \leq 1$ and $\bar{F}(x, y)(t)=1$ if $t>1$, then $\bar{F}$ is a probabilistic pseudometric on $X$ and $\tau_{\bar{F}}=\tau_{F}$. Hence, we may assume that $F_{n}(x, y)(t)=1$, for all $n$, if $t>1$. For $x, y \in X$, define $F(x, y)$ on $\mathbb{R}$ by $F(x, y)(t)=0$ if $t \leq 0$ and $F(x, y)(t)=\inf _{n}\left[(1 / n) F_{n}(x, y)\right](t)$ if $t>0$. Clearly, $F(x, y)$ is increasing and $F(x, y)(t)=1$ if $t>1$. Also, $F(x, y)$ is left continuous. In fact, let $F(x, y)(t)>\theta>0$ and choose $n$ such that $(n+1) t>1$. There exists $0<s_{1}<t$ such that $F_{k}(x, y)\left(k s_{1}\right)>\theta$ for $k=1, \ldots, n$. Choose $s_{1}<s<t$ such that $(n+1) s>1$. Now, $F_{m}(x, y)(m s)=1$ if $m>n$. Thus

$$
F(x, y)(s)=\min _{1 \leq k \leq n}\left[\frac{1}{k} F_{k}(x, y)\right](s)>\theta,
$$

which proves that $F(x, y)$ is in $\mathbb{R}_{\phi}^{+}$. It is clear that $F(x, x)=\overline{0}$. We need to prove that $F$ satisfies the triangle inequality. So assume that $F(x, y)\left(t_{1}\right) \wedge F(y, z)\left(t_{2}\right)>\theta>0$. If $m$ is such that $(m+1)\left(t_{1}+t_{2}\right)>1$, then

$$
F(x, z)\left(t_{1}+t_{2}\right)=\min _{1 \leq k \leq m} F_{k}(x, z)\left(k\left(t_{1}+t_{2}\right)\right) .
$$

Since

$$
F_{k}(x, z)\left(k\left(t_{1}+t_{2}\right)\right) \geq F_{k}(x, y)\left(k t_{1}\right) \wedge F_{k}(y, z)\left(k t_{2}\right)>\theta
$$

it follows that $F(x, z)\left(t_{1}+t_{2}\right)>\theta$, and so $F$ satisfies the triangle inequality. We will finish the proof by showing that $\tau_{F}=\bigvee \tau_{F_{n}}$. To see this, we first observe that $(1 / n) F_{n} \preceq F$, which implies that $\tau_{F_{n}}=\tau_{(1 / n) F_{n}} \leq \tau_{F}$, and so $\tau_{o}=\bigvee_{n} \tau_{(1 / n) F_{n}} \leq \tau_{F}$. On the other hand, let $N_{x}^{\tau_{F}}(A)>\theta$ and choose $\epsilon>0$ such that $N_{x}^{\tau_{F}}(A)>\theta+\epsilon$. Let $t>0$ be such that $1-$ $\sup _{y \notin A} F(y, x)(t)>\theta+\epsilon$. If $(m+1) t>1$, then

$$
F(y, z)(t)=\min _{1 \leq k \leq m} F_{k}(y, z)(k t)
$$


Let $A_{k}=\left\{y: F_{k}(y, x)(k t) \geq 1-\theta-\epsilon\right\}$. Then

$$
N_{x}^{\tau_{o}}\left(A_{k}\right) \geq N_{x}^{\tau_{F_{k}}}\left(A_{k}\right) \geq 1-\sup _{z \notin A_{k}} F_{k}(z, x)(k t) \geq \theta+\epsilon>\theta
$$

and $\bigcap_{k=1}^{m} A_{k} \subset A$. Hence, $N_{x}^{\tau_{o}}(A) \geq \min _{1 \leq k \leq m} N_{x}^{\tau_{o}}\left(A_{k}\right)>\theta$. This proves that $\tau_{F} \leq \tau_{o}$ and the result follows.

Theorem 3.10. Let $f: X \rightarrow Y$ be a function and let $F$ be a probabilistic pseudometric on $Y$. Then the function

$$
f^{-1}(F): X^{2} \longrightarrow \mathbb{R}_{\phi}^{+1}, \quad f^{-1}(F)(x, y)=F(f(x), f(y))
$$

is a probabilistic pseudometric on $X$ and $\tau_{f^{-1}(F)}=f^{-1}\left(\tau_{F}\right)$.

Proof. It follows easily that $f^{1}(F)$ is a probabilistic pseudometric on $X$. Let $x \in X$ and $B \subset X$. If $D=Y \backslash f\left(B^{c}\right)$, then

$$
\begin{aligned}
N_{x}^{\tau_{f-1}(F)}(B) & =\inf _{t>0}\left[1-\sup _{y \notin B} F(f(y), f(x))(t)\right] \\
& =\inf _{t>0}\left[1-\sup _{z \in D^{c}} F(z, f(x))(t)\right] \\
& =N_{f(x)}^{\tau_{F}}(D)=N_{x}^{f^{-1}\left(\tau_{F}\right)}(B),
\end{aligned}
$$

which clearly completes the proof.

Corollary 3.11. If $F$ is a probabilistic pseudometric on a set $X$ and $Y \subset X$, then $\left.\tau_{F}\right|_{Y}$ is induced by the probabilistic pseudometric $G=\left.F\right|_{Y \times Y}, G(x, y)=F(x, y)$.

Corollary 3.12. If $\left(X_{n}, \tau_{n}\right)$ is a sequence of pseudometrizable fuzzifying topological spaces, then the Cartesian product $(X, \tau)=\left(\prod X_{n}, \prod \tau_{n}\right)$ is pseudometrizable.

Proof. Let $F_{n}$ be a probabilistic pseudometric on $X_{n}$ inducing $\tau_{n}$. If $G_{n}=\pi_{n}^{-1}\left(F_{n}\right)$, then $\tau_{G_{n}}=\pi_{n}^{-1}\left(\tau_{n}\right)$, and so $\tau=\bigvee_{n} \pi_{n}^{-1}\left(\tau_{n}\right)$ is pseudometrizable.

\section{Level proximities}

Let $\delta$ be a fuzzifying proximity on a set $X$. For each $0<d \leq 1$, let $\delta^{d}$ be the binary relation on $2^{X}$ defined by $A \delta^{d} B$ if and only if $\delta(A, B) \geq d$. It is easy to see that $\delta^{d}$ is a classical proximity on $X$. We will show that the classical topology $\sigma_{d}$ induced by $\delta^{d}$ coincides with $\tau^{1-d}$. In fact, let $x \in A \in \sigma_{d}$. Then, $x$ is not in the $\sigma_{d}$-closure of $A^{c}$, which implies that $x \delta^{d} A^{c}$, that is, $\delta\left(x, A^{c}\right)<d$, and so $N_{x}^{\tau}(A)=1-\delta\left(x, A^{c}\right)>1-d$. This proves that $A \in \tau^{1-d}$. Conversely, if $x \in B \in \tau^{1-d}$, then $N_{x}^{\tau}(A)>1-d$, and thus $\delta\left(x, A^{c}\right)<d$, which implies that $x$ is not in the $\sigma_{d}$-closure of $B^{c}$. Hence $B^{c}$ is $\sigma_{d}$-closed, and so $B$ is $\sigma_{d}$-open. 
Theorem 4.1. If $\delta$ is a fuzzifying proximity on a set $X$ and $0<d \leq 1$, then

$$
\delta^{d}=\bigvee_{0<\theta<d} \delta^{\theta}
$$

Proof. If $0<\theta<d$, then $\delta^{\theta}$ is coarser than $\delta^{d}$, and so $\delta_{o}=\bigvee_{0<\theta<d} \delta^{\theta}$ is coarser than $\delta^{d}$. On the other hand, let $A \delta_{o} B$. Since $\delta_{o}$ is finer than $\delta^{\theta}$ (for $0<\theta<d$ ), we have that $A \delta^{\theta} B$ and so $\delta(A, B) \geq \theta$, for each $0<\theta<d$, which implies that $\delta(A, B) \geq d$, that is, $A \delta^{d} B$. So $\delta_{o}$ is finer than $\delta^{d}$ and the result follows.

Theorem 4.2. For a family $\left\{\gamma_{d}: 0<d \leq 1\right\}$ of classical proximities on a set $X$, the following are equivalent.

(1) There exists a fuzzifying proximity $\delta$ on $X$ such that $\delta^{d}=\gamma_{d}$ for all $d$.

(2) $\gamma_{d}=\bigvee_{0<\theta<d} \gamma_{\theta}$ for each $0<d \leq 1$.

Proof. In view of the preceding theorem, (1) implies (2). Assume now that (2) is satisfied and define $\delta$ on $2^{X} \times 2^{X}$ by $\delta(A, B)=\sup \left\{d: A \gamma_{d} B\right\}$ (the supremum over the empty family is taken to be zero). It is clear that $\delta(A, B)=1$ if the $A, B$ are not disjoint. Also, $\delta(A, B)=\delta(A, B)$ and $\delta\left(A, B \geq \delta\left(A_{1}, B_{1}\right)\right.$ if $A_{1} \subset A, B_{1} \subset B$. Let now $\delta(A, B)<d<1$. Then $A y_{d} B$, and so there exists a subset $D$ of $X$ such that $A y_{d} D$ and $D^{c} y_{d} B$. Since $A y_{d} D$, we have that $\delta(A, D) \leq d$. Similarly $\delta\left(D^{c}, B\right) \leq d$, and so $\inf \left\{\delta(A, D) \wedge \delta\left(D^{c}, B\right)\right\} \leq \delta(A, B)$. On the other hand, if $\delta(A, D) \wedge \delta\left(D^{c}, B\right)<\theta<1$, then $A \subset D^{c}$, and so $\delta(A, B) \leq \delta\left(D^{c}, B\right)<$ $\theta$. This proves that $\delta$ is a fuzzifying proximity on $X$. We will finish the proof by showing that $\delta^{d}=\gamma_{d}$ for all $d$. Indeed, if $A \gamma_{d} B$, then $\delta(A, B) \geq d$, that is, $A \delta^{d} B$. On the other hand, let $A \delta^{d} B$ and let $\left(A_{i}\right),\left(B_{j}\right)$ be finite families of subsets of $X$ with $A=\bigcup_{i}, B=\bigcup B_{j}$. Since $\delta(A, B)=\bigvee_{i, j} \delta\left(A_{i}, B_{j}\right) \geq d$, there exists a pair $(i, j)$ such that $\delta\left(A_{i}, B_{j}\right) \geq d$. If now $0<\theta<d$, then there exists $r>\theta$ with $A_{i} \gamma_{r} B_{j}$, and so $A_{i} \gamma_{\theta} B_{j}$. This proves that $A \gamma_{d} B$ since $\gamma_{d}=\bigvee_{0<\theta<d} \gamma_{\theta}$. This completes the proof.

Theorem 4.3. Let $\left(X, \delta_{1}\right),\left(Y \delta_{2}\right)$ be fuzzifying proximity spaces and let $f: X \rightarrow Y$ be a function. Then $f$ is proximally continuous if and only if $f:\left(X, \delta_{1}^{d}\right) \rightarrow\left(Y, \delta_{2}^{d}\right)$ is proximally continuous for each $0<d \leq 1$.

Proof. It follows immediately from the definitions.

THEOREM 4.4. Let $\left(X_{\lambda}, \delta_{\lambda}\right)_{\lambda \in \Lambda}$ be a family of fuzzifying proximity spaces and let $(X, \delta)=$ $\left(\prod X_{\lambda}, \prod \delta_{\lambda}\right)$ be the product fuzzifying proximity space. Then $\delta^{d}=\prod \delta_{\lambda}^{d}$ for all $0<d \leq 1$.

Proof. Since each projection $\pi_{\lambda}:\left(X, \delta^{d}\right) \rightarrow\left(X_{\lambda}, \delta_{\lambda}^{d}\right)$ is proximally continuous, it follows that $\delta^{d}$ is finer than $\sigma=\prod \delta_{\lambda}^{d}$. On the other hand, let $A \sigma B$. We need to show that $\delta(A, B) \geq$ $d$. In fact, let $\left(A_{i}\right),\left(B_{j}\right)$ be finite families of subsets of $X$ such that $A=\bigcup A_{i}, B=\bigcup B_{j}$. Since $A \sigma B$ and $\sigma=\bigvee_{\lambda} \pi_{\lambda}^{-1}\left(\delta_{\lambda}^{d}\right)$, there exists a pair $(i, j)$ such that $A_{i} \pi_{\lambda}^{-1}\left(\delta^{d}\right) B_{j}$, that is, $\delta_{\lambda}\left(\pi_{\lambda}\left(A_{i}\right), \pi_{\lambda}\left(B_{j}\right)\right) \geq d$. In view of $[4$, Theorem 8.9], we conclude that $\delta(A, B) \geq d$. Hence, $\sigma=\delta^{d}$ and the proof is complete.

We have the following easily established theorem.

TheOREM 4.5. Let $(Y, \delta)$ be a fuzzifying proximity space and let $f: X \rightarrow Y$. Then $f^{-1}(\delta)^{d}=$ $f^{-1}\left(\delta^{d}\right)$ for each $0<d \leq 1$. 
Theorem 4.6. Let $\left(\delta_{\lambda}\right)_{\lambda \in \Lambda}$ be a family of fuzzifying proximities on a set $X$ and $\delta=\vee_{\lambda} \delta_{\lambda}$. Then $\delta^{d}=\bigvee_{\lambda} \delta_{\lambda}^{d}$ for each $0<d \leq 1$.

Proof. Let $\sigma=\bigvee_{\lambda} \delta_{\lambda}^{d}$. Since $\delta$ is finer than each $\delta_{\lambda}$, it follows that $\delta^{d}$ is finer than each $\delta_{\lambda}^{d}$, and so $\delta^{d}$ is finer than $\sigma$. On the other hand, let $A \sigma B$ and let $\left(A_{i}\right),\left(B_{j}\right)$ be finite families of subsets of $X$ such that $A=\bigcup A_{i}, B=\bigcup B_{j}$. There exists a pair $(i, j)$ such that $A_{i} \sigma B_{j}$. Since $\sigma$ is finer than each $\delta_{\lambda}^{d}$, we have that $A_{i} \delta_{\lambda}^{d} B_{j}$, that is, $\delta_{\lambda}\left(A_{i}, B_{j}\right) \geq d$. In view of [4, Theorem 8.10], we get that $\delta(A, B) \geq d$, that is, $A \delta^{d} B$. So $\sigma$ is finer than $\delta^{d}$ and the proof is complete.

\section{Completely regular fuzzifying spaces}

Definition 5.1. A fuzzifying topological space $(X, \tau)$ is called completely regular if each of the classical level topologies $\tau^{d}, 0 \leq d<1$, is completely regular.

Definition 5.2. A fuzzifying proximity $\delta$ on a set $X$ is said to be compatible with a fuzzifying topology $\tau$ if $\tau$ coincides with the fuzzifying topology $\tau_{\delta}$ induced by $\delta$.

We have the following easily established theorem.

Theorem 5.3. Subspaces and Cartesian products of completely regular fuzzifying spaces are completely regular.

Theorem 5.4. Let $(X, \tau)$ be a completely regular fuzzifying topological space and define $\delta=\delta(\tau): 2^{X} \times 2^{X} \rightarrow[0,1]$ by

$$
\delta(A, B)=1-\sup \left\{d: 0 \leq d<1, \exists f:\left(X, \tau^{d}\right) \longrightarrow[0,1] \text { continuous } f(A)=0, f(B)=1\right\} .
$$

Then, (1) $\delta$ is a fuzzifying proximity on $X$ compatible with $\tau$;

(2) if $\delta_{1}$ is any fuzzifying proximity on $X$ compatible with $\tau$, then $\delta$ is finer than $\delta_{1}$.

Proof. It is easy to see that $\delta$ satisfies (FP1), (FP2), (FP3), and (FP5). We will prove that $\delta$ satisfies (FP4). Let

$$
\alpha=\inf \left\{\delta(A, D) \vee \delta\left(D^{c}, B\right): D \subset X\right\} .
$$

If $\delta(A, D) \vee \delta\left(D^{c}, B\right)<\theta$, then $A \subset D^{c}$, and so $\delta(A, B) \leq \delta\left(D^{c}, B\right)<\theta$, which proves that $\delta(A, B) \leq \alpha$. On the other hand, assume that $\delta(A, B)<r<1$. There exist a $d, 1-r<d<1$, and $f: X \rightarrow[0,1] \tau^{d}$-continuous such that $f(A)=0, f(B)=1$. Let $D=\{x \in X: 1 / 2 \leq$ $f(x) \leq 1\}$ and define $h_{1}, h_{2}:[0,1] \rightarrow[0,1], h_{1}(t)=2 t, h_{2}(t)=0$ if $0 \leq t \leq 1 / 2$ and $h_{1}(t)=$ $1, h_{2}(t)=2 t-1$ if $1 / 2<t \leq 1$. If $g_{i}=h_{i} \circ f, i=1,2$, then $g_{1}(A)=0, g_{1}(D)=1, g_{2}\left(D^{c}\right)=0$, $g_{2}(B)=1$. Thus, $\delta(A, D) \leq 1-d<r, \delta\left(D^{c}, B\right)<r$, which proves that $\alpha \leq \delta(A, B)$. Hence, $\delta$ is a fuzzifying proximity on $X$. We need to show that $\tau=\tau_{\delta}$. So, let $\tau(A)>\theta>0$. Since $\tau^{\theta}$ is completely regular, given $x \in A$, there exist $f_{x}: X \rightarrow[0,1], \tau^{\theta}$-continuous, $f_{x}(x)=$ $0, f_{x}\left(A^{c}\right)=1$. Thus $\delta\left(x, A^{c}\right) \leq 1-\theta$, and so $N_{x}^{\tau_{\delta}}(A)=1-\delta\left(x, A^{c}\right) \geq \theta$. It follows that $\tau_{\delta}(A)=\inf _{x \in A} N_{x}^{\tau_{\delta}}(A) \geq \theta$, which proves that $\tau_{\delta} \geq \tau$. On the other hand, assume that $\tau_{\delta}(A)>r>0$. If $x \in A$, then $\delta\left(x, A^{c}\right)=1-N_{x}^{\tau_{\delta}}(A)<1-r$, and therefore there exist a $d$, $0<1-d<1-r$ and $f: X \rightarrow[0,1] \tau^{d}$-continuous such that $f(x)=0, f\left(A^{c}\right)=1$. The set 
$G=\{y: f(y)<1 / 2\}$ is in $\tau^{d}$ and $x \in G \subset A$. Thus,

$$
N_{x}^{\tau}(A) \geq N_{x}^{\tau}(G) \geq d>r .
$$

This proves that $\tau(A) \geq r$ and so $\tau \geq \tau_{\delta}$, which completes the proof of (1).

Let $\delta_{1}$ be a fuzzifying proximity on $X$ compatible with $\tau$ and let $A, B$ be subsets of $X$ with $\delta_{1}(A, B)<\theta<1$. If $d=1-\theta$, then $\delta_{1}^{\theta}$ is compatible with $\tau^{d}$. Since $A \delta_{1}^{\theta} B$, there exists (by $\left[8\right.$, Remark 3.15]) an $f: X \rightarrow[0,1] \tau_{d}$-continuous, with $f(A)=0, f(B)=1$, and so $\delta(A, B) \leq 1-d=\theta$, which proves that $\delta(A, B) \leq \delta_{1}(A, B)$, and therefore $\delta$ is finer than $\delta_{1}$. This completes the proof.

Theorem 5.5. For a fuzzifying topological space $(X, \tau)$, the following are equivalent.

(1) $(X, \tau)$ is completely regular.

(2) There exists a fuzzifying proximity $\delta$ on $X$ compatible with $\tau$.

(3) $(X, \tau)$ is fuzzy uniformizable, that is, there exists a fuzzy uniformity $U$ on $X$ such that $\tau$ coincides with the fuzzifying topology $\tau$ 证 inced by $\boldsymbol{U}$.

Proof. By [5], (2) is equivalent to (3). Also (1) implies (2) in view of the preceding theorem. Assume now that $\tau=\tau_{\delta}$ for some fuzzifying proximity $\delta$. For each $0<d \leq 1, \delta^{d}$ is a classical proximity compatible with $\tau^{1-d}$, and so $\tau^{1-d}$ is completely regular. This completes the proof.

Theorem 5.6. Every pseudometrizable fuzzy topological space $(X, \tau)$ is completely regular.

Proof. If $\tau$ is pseudometrizable, then each $\tau^{d}, 0 \leq d<1$, is pseudometrizable, and hence $\tau^{d}$ is completely regular.

Theorem 5.7. For a fuzzifying topological space $(X, \tau)$, the following are equivalent.

(1) $(X, \tau)$ is completely regular.

(2) If $\mathscr{F}=\mathscr{F}_{\tau}$ is the family of all probabilistic pseudometrics on $X$ which are $\tau \times \tau$ continuous as functions from $X^{2}$ to $\mathbb{R}_{\phi}^{+}$, then $\tau=\tau \mathscr{F}_{\tau}$.

(3) There exists a family $\mathscr{F}_{\mathscr{F}}$ of probabilistic pseudometrics on $X$ such that $\tau=\tau_{\mathscr{F}}$.

Proof. (1) $\Rightarrow(2)$. For each $F \in \mathscr{F}_{\tau}$, we have that $\tau_{F} \leq \tau$ (by Theorem 3.2), and so $\tau_{\mathscr{F}_{\tau}} \leq \tau$. Let now $A \subset X$ and $x_{o} \in X$ with $N_{x_{0}}^{\tau}(A)>\theta>0$. Since $\tau^{\theta}$ is completely regular, there exists a $\tau^{\theta}$-continuous function $f$ from $X$ to $[0,1]$ such that $f\left(x_{o}\right)=0, f\left(A^{c}\right)=1$. For $x, y \in X$, define $F(x, y)$ on $\mathbb{R}$ by

$$
F(x, y)(t)= \begin{cases}0 & \text { if } t \leq 0 \\ 1-\theta & \text { if }|f(x)-f(y)| \geq t>0 \\ 1 & \text { if }|f(x)-f(y)|<t .\end{cases}
$$

Clearly, $F(x, y)=F(y, x) \in \mathbb{R}_{\phi}^{+}$and $F(x, x)=\overline{0}$. We will prove that $F$ satisfies the triangle inequality. So, assume that $F(x, y)\left(t_{1}\right) \wedge F(y, z)\left(t_{2}\right)>F(x, z)\left(t_{1}+t_{2}\right)$. Then, $t_{1}, t_{2}>0$, $F(x, z)\left(t_{1}+t_{2}\right)=1-\theta, F(x, y)\left(t_{1}\right)=F(y, z)\left(t_{2}\right)=1$. Thus, $t_{1}>|f(x)-f(y)|, t_{2}>$ $|f(y)-f(z)|$, and hence $|f(x)-f(z)|<t_{1}+t_{2}$, which implies that $F(x, z)\left(t_{1}+t_{2}\right)=1$, a contradiction. So $F$ is a probabilistic pseudometric on $X$. Next we show that $F$ is $\tau \times \tau$ continuous, or equivalently that $\tau_{F} \leq \tau$. So assume that $N_{x}^{\tau_{F}}(B)>r>0$. Let $\theta_{1}>r$ be such 
that $N_{x}^{\tau_{F}}(B)>\theta_{1}$. Choose $t>0$ such that $1-\sup _{y \notin B} F(x, y)(t)>\theta_{1}$, and so $F(x, y)(t)=$ $1-\theta$ and $|f(x)-f(y)| \geq t$ if $y \notin B$. Thus, $\{y:|f(x)-f(y)|<t\} \subset B$. This shows that $B$ is a $\tau^{\theta}$-neighborhood of $x$. As $r<\theta, B$ is a $\tau^{r}$-neighborhood of $x$, that is, $N_{x}^{\tau}(B)>r$, and so $\tau_{F} \leq \tau$. Finally if $y \notin A$, then $\left|f(y)-f\left(x_{o}\right)\right|=1$, and so $F\left(y, x_{o}\right)(1 / 2)=1-\theta$, which implies that

$$
N_{x_{o}}^{\tau \mathscr{F}}(A) \geq N_{x_{o}}^{\tau_{F}}(A) \geq 1-\sup _{y \notin A} F\left(y, x_{o}\right)\left(\frac{1}{2}\right) \geq \theta .
$$

This shows that $N_{x_{0}}^{\tau_{\mathscr{F}}} \geq N_{x_{0}}^{\tau}$, and so $\tau \leq \tau_{\mathscr{F}}$, which completes the proof of the implication $(1) \Rightarrow(2)$.

(3) $\Rightarrow(1)$. Assume that $\tau=\tau_{\mathscr{F}}$ for some family $\mathscr{F}$ of probabilistic pseudometrics on $X$. For each $F \in \mathscr{F}, \tau_{F}$ is completely regular and so $\tau_{\mathscr{F}}$ is completely regular since $\tau_{\mathscr{F}_{F}}^{d}=$ $\bigvee_{F \in \mathscr{F}} \tau_{F}^{d}$ for each $0 \leq d<1$. Hence the result follows.

We will denote by $[0,1]_{\phi}$ the subspace of $\mathbb{R}_{\phi}^{+}$consisting of all $u \in \mathbb{R}_{\phi}^{+}$with $u(t)=1$ if $t>1$.

Theorem 5.8. A fuzzifying topological space $(X, \tau)$ is completely regular if and only if the following condition is satisfied. If $N_{x_{0}}(A)>\theta>0$, then there exists $f: X \rightarrow[0,1]_{\phi}$ continuous such that $f\left(x_{o}\right)=\overline{0}$ and $f(y)(t)=1-\theta$ if $y \notin A$ and $0<t<1$.

Proof. Assume that $(X, \tau)$ is completely regular and let $N_{x_{o}}(A)>\theta>0$. Since $\tau^{\theta}$ is completely regular, there exists $h:\left(X, \tau^{\theta}\right) \rightarrow[0,1]$ continuous, $h\left(x_{0}\right)=0, h(y)=1$ if $y \notin A$. For $x, y \in X$, define $F(x, y)$ on $\mathbb{R}$ by

$$
F(x, y)(t)= \begin{cases}0 & \text { if } t \leq 0 \\ 1-\theta & \text { if }\left|h(x)-h\left(x_{0}\right)\right| \geq t>0 \\ 1 & \text { if }\left|h(x)-h\left(x_{0}\right)\right|<t\end{cases}
$$

Clearly, $F(x, y) \in[0,1]_{\phi}$. Also, $F(x, z) \preceq F(x, y) \oplus F(y, z)$. In fact, assume that $F(x, y)$ $\left(t_{1}\right) \wedge F(y, z)\left(t_{2}\right)>r>F(x, z)\left(t_{1}+t_{2}\right)$. Then $t_{1}, t_{2}>0, F(x, y)\left(t_{1}\right)=F(y, z)\left(t_{2}\right)=1$. Now, $|h(x)-h(y)|<t_{1},|h(y)-h(z)|<t_{2}$, and so $|h(x)-h(z)|<t_{1}+t_{2}$, which implies that $F(x, z)\left(t_{1}+t_{2}\right)=1$, a contradiction. So $F$ is a probabilistic pseudometric. Moreover, $F$ is $\tau \times \tau$ continuous, or equivalently $\tau_{F} \leq \tau$. In fact, let $N_{x}^{\tau_{F}}(B)>r>0$. There exists a $t>0$ such that $1-\sup _{z \notin B} F(z, x)(t)>r$. If $z \notin B$, then $F(z, x)(t)<1-r<1$, and so $F(z, x)(t)=$ $1-\theta<1-r$, that is, $r<\theta$, and $|h(z)-h(x)| \geq t$. Hence

$$
M=\{z:|h(z)-h(x)|<t\} \subset B .
$$

The set $M$ is a $\tau^{\theta}$-neighborhood of $x$, and hence a $\tau^{r}$-neighborhood, that is, $N_{x}^{\tau}(B)>r$. Thus $\tau \geq \tau_{F}$. Finally, define $f: X \rightarrow[0,1]_{\phi}, f(y)=F\left(y, x_{o}\right)$. Then $f$ is $\tau$-continuous, $f\left(x_{o}\right)=\overline{0}$. For $y \notin A$ and $0<t<1$, we have that $f(y)(t)=F\left(y, x_{o}\right)(t)=1-\theta$ (since $\mid h(x)-h\left(x_{o} \mid=1 \geq t\right)$. Conversely, assume that the condition is satisfied and let $\mathscr{F}_{\mathrm{f}}$ be the family of all $\tau \times \tau$ continuous pseudometrics on $X$. Then $\tau_{\mathscr{F}} \leq \tau$. Let $N_{x_{0}}^{\tau}(A)>\theta$. There 
exists a $\theta_{1}>\theta$ such that $N_{\chi_{o}}^{\tau}(A)>\theta_{1}$. By our hypothesis, there exists $f: X \rightarrow[0,1]_{\phi}$ continuous such that $f\left(x_{o}\right)=\overline{0}$ and $f(y)(t)=1-\theta_{1}$ if $y \notin A$ and $0<t<1$. Define $F(x, y)$ $=D(f(x), f(y))$. Then $F$ is $\tau \times \tau$ continuous and

$$
\begin{aligned}
N_{x_{o}}^{\tau_{F}}(A) & \geq N_{x_{o}}^{\tau_{F}}(A) \geq 1-\sup _{y \notin A} F\left(x_{o}, y\right)(1) \\
& =1-\sup _{y \notin A} D(\overline{0}, f(y))(1) \\
& =1-\sup _{y \notin A} f(y)(1) \geq \theta_{1}>\theta .
\end{aligned}
$$

Thus $N_{x_{o}}^{\tau \mathscr{F}}(A) \geq N_{x_{o}}^{\tau}(A)$, for every subset $A$ of $X$, and so $\tau \leq \tau_{\mathscr{F}}$. Therefore, $\tau=\tau_{\mathscr{F}}$, and so $\tau$ is completely regular.

For a fuzzifying topological space $X$, we will denote by $C\left(X,[0,1]_{\phi}\right)$ the family of all continuous functions from $X$ to $[0,1]_{\phi}$.

THeOREM 5.9. A fuzzifying topological space $(X, \tau)$ is completely regular if and only if $\tau$ coincides with the weakest of all fuzzifying topologies $\tau_{1}$ on $X$ for which each $f \in C\left(X,[0,1]_{\phi}\right)$ is continuous.

Proof. Assume that $(X, \tau)$ is completely regular and let $\tau_{1}$ be the weakest of all fuzzifying topologies on $X$ for which each $f \in C\left(X,[0,1]_{\phi}\right)$ is continuous. Clearly $\tau_{1} \leq \tau$. On the other hand, let $\tau_{2}$ be a fuzzifying topology on $X$ for which each $f \in C\left(X,[0,1]_{\phi}\right)$ is continuous. Let $N_{x}^{\tau}(A)>\theta>0$. In view of the preceding theorem, there exists an $f \in$ $C\left(X,[0,1]_{\phi}\right)$ such that $f(x)=\overline{0}, f(y)(t)=1-\theta$ if $y \notin A$ and $0<t<1$. Let

$$
G=\left\{u \in \mathbb{R}_{\phi}^{+}: D(f(x), u)\left(\frac{1}{2}\right)=u\left(\frac{1}{2}\right)>1-\theta\right\}
$$

Then

$$
N_{\overline{0}}(G) \geq 1-\sup _{u \notin G} D(f(x), u)\left(\frac{1}{2}\right) \geq \theta
$$

Since $f$ is $\tau_{2}$-continuous, we have that $N_{x}^{\tau_{2}}\left(f^{-1}(G)\right) \geq \theta$. But $f^{-1}(G) \subset A$ since, for $y \notin A$, we have that $f(y)(1 / 2)=1-\theta$. Thus $N_{x}^{\tau_{2}}(A) \geq \theta$. This proves that $N_{x}^{\tau_{2}}(A) \geq N_{x}^{\tau}(A)$, for every subset $A$ of $X$, and so $\tau_{2} \geq \tau$. This clearly proves that $\tau_{1}=\tau$. Conversely, assume that $\tau_{1}=\tau$. If $\sigma$ is the usual fuzzifying topology of $\mathbb{R}_{\phi}^{+}$, then

$$
\tau=\tau_{1}=\bigvee_{f \in C\left(X,[0,1]_{\phi}\right)} f^{-1}(\sigma)
$$

Since $\sigma$ is completely regular, each $f^{-1}(\sigma)$ is completely regular, and so $\tau$ is completely regular. This completes the proof. 


\section{References}

[1] U. Höhle, Upper semicontinuous fuzzy sets and applications, J. Math. Anal. Appl. 78 (1980), no. 2, 659-673.

[2] _ Probabilistic metrization of fuzzy uniformities, Fuzzy Sets and Systems 8 (1982), no. 1, 63-69.

[3] Characterization of L-topologies by L-valued neighborhoods, Mathematics of Fuzzy Sets: Logic, Topology, and Measure Theory, Handb. Fuzzy Sets Ser., vol. 3, Kluwer Academic, Massachusetts, 1999, pp. 389-432.

[4] A. K. Katsaras and C. G. Petalas, Fuzzifying topologies and fuzzifying proximities, J. Fuzzy Math. 11 (2003), no. 2, 411-436.

[5] _ Fuzzifying syntopogenous structures, J. Fuzzy Math. 12 (2004), no. 1, 77-108.

[6] _ On fuzzifying topological spaces, J. Fuzzy Math. (2005).

[7] R. Lowen, Convergence in fuzzy topological spaces, General Topology Appl. 10 (1979), no. 2, $147-160$.

[8] S. A. Naimpally and B. D. Warrack, Proximity Spaces, Cambridge Tracts in Mathematics and Mathematical Physics, no. 59, Cambridge University Press, London, 1970.

[9] M. S. Ying, A new approach for fuzzy topology. I, Fuzzy Sets and Systems 39 (1991), no. 3, 303321.

[10] A new approach for fuzzy topology. II, Fuzzy Sets and Systems 47 (1992), no. 2, 221232.

[11] A new approach for fuzzy topology. III, Fuzzy Sets and Systems 55 (1993), no. 2, 193207.

[12] On the method of neighborhood systems in fuzzy topology, Fuzzy Sets and Systems 68 (1994), no. 2, 227-238.

[13] D. Zhang and L. Xu, Categories isomorphic to FNS, Fuzzy Sets and Systems 104 (1999), no. 3, 373-380.

A. K. Katsaras: Department of Mathematics, School of Natural Sciences, University of Ioannina, 45110 Ioannina, Greece

E-mail address: akatsar@cc.uoi.gr 


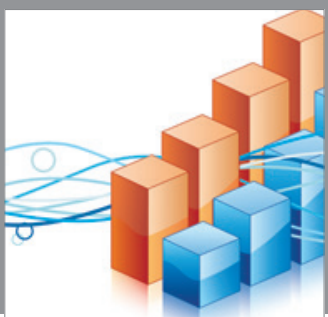

Advances in

Operations Research

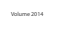

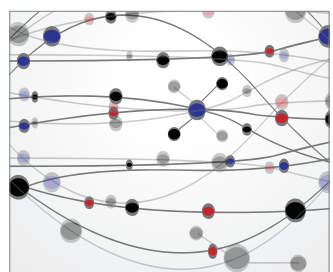

\section{The Scientific} World Journal
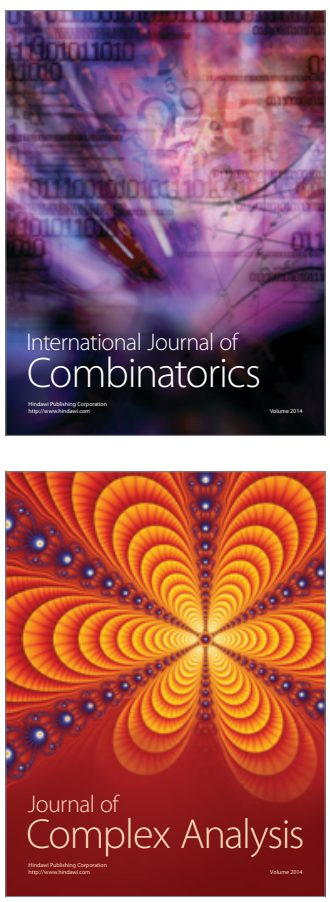

International Journal of

Mathematics and

Mathematical

Sciences
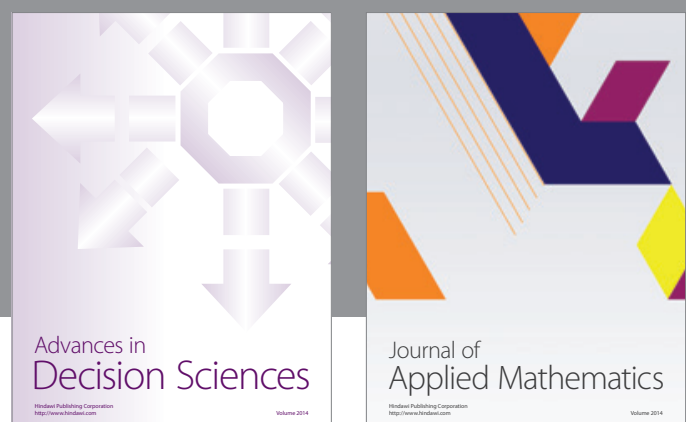

Journal of

Applied Mathematics
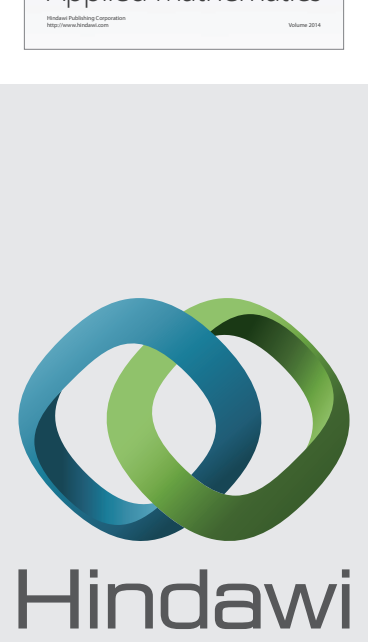

Submit your manuscripts at http://www.hindawi.com
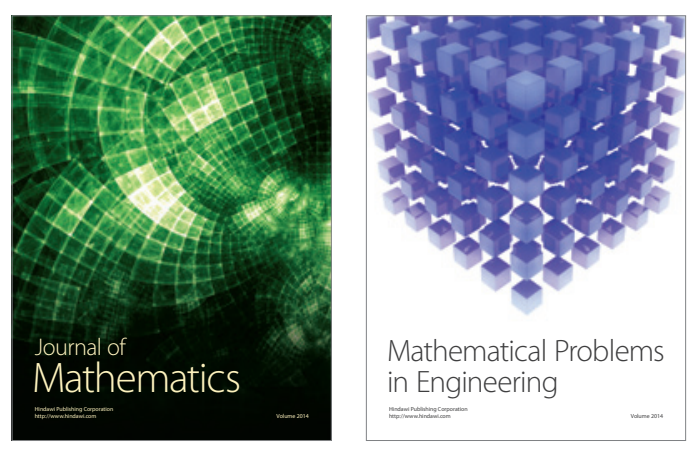

Mathematical Problems in Engineering
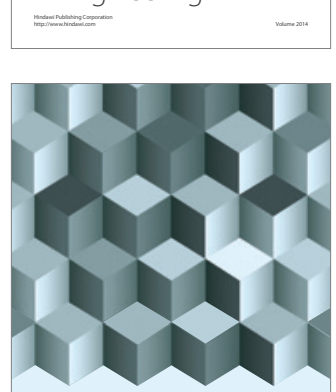

Journal of

Function Spaces
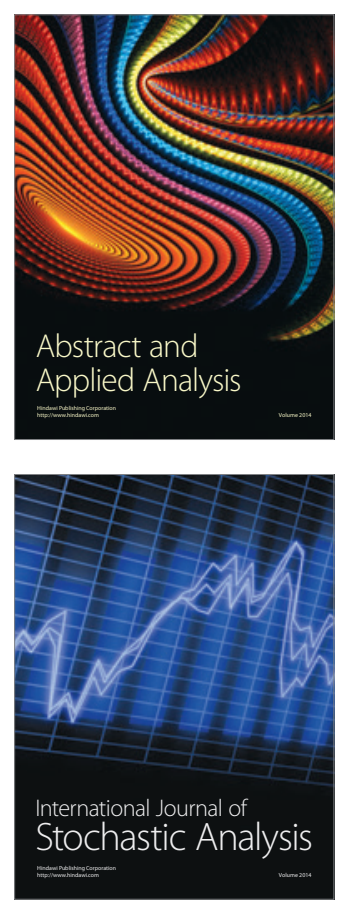

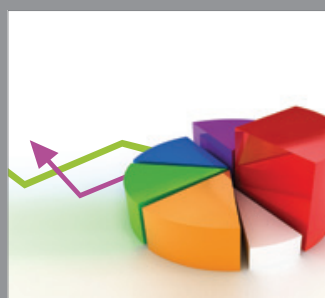

ournal of

Probability and Statistics

Promensencen
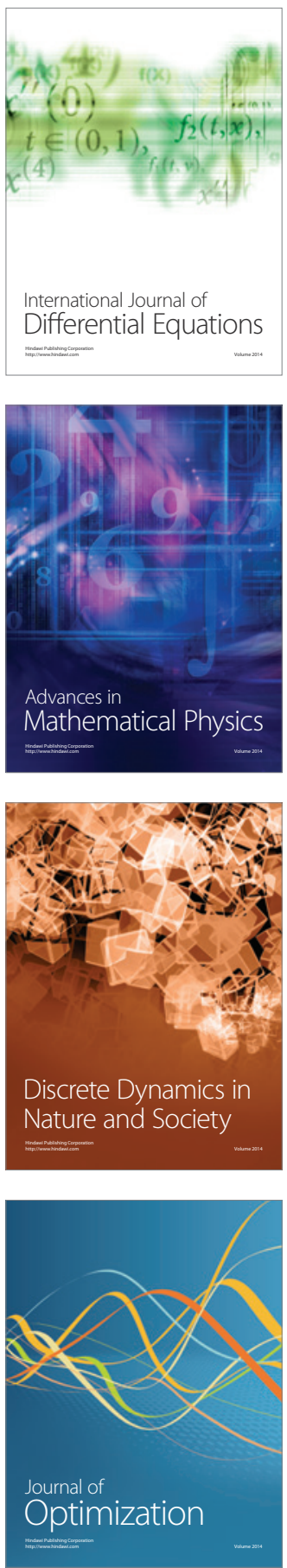